4. Shields CL, Shields JA, Augsburger JJ. Choroidal osteoma. Surv Ophthalmol 1988;33:17-27.

5. Sanborn GE, Augsburger JJ, Shields JA. Spontaneous regression of bilateral retinoblastoma. $\mathrm{Br} \mathrm{J}$ Ophthalmol 1982;66:685.

6. Munier F, Zografos L, Schnyder P. Idiopathic sclerochoroidal calcification: new observations. Eur $\mathbf{J}$ Ophthalmol 1991;1:167-72.

7. Sivalingam A, Shields CL, Shields JA, McNamara JA, Jampol LM, Wood WJ, Daubert G. Idiopathic sclerochoroidal calcification. Ophthalmology 1991; 98:720-4.

Sir,

\section{Bilateral Buried Optic Nerve Drusen Presenting with Central Retinal Artery Occlusion at High Altitude \\ Retinal ischaemia at high altitude is well recog-} nised $^{1,2}$ and may have disastrous consequences.

\section{Case Report}

A 48-year-old man presented with a sudden loss of vision in his left eye during descent to landing at Heathrow airport. Five years previously a similar incident occurred in his right eye during a skiing holiday, after descent from high altitude, resulting in hand movements vision. $\mathrm{He}$ was otherwise fit and intermittently took $300 \mathrm{mg}$ aspirin o.d. as prophylaxis.

The vision was hand movements in both eyes; both anterior segments were normal and both pupils reacted slowly to light. A left central artery occlusion (CRAO) was diagnosed (Fig. 1) and optic

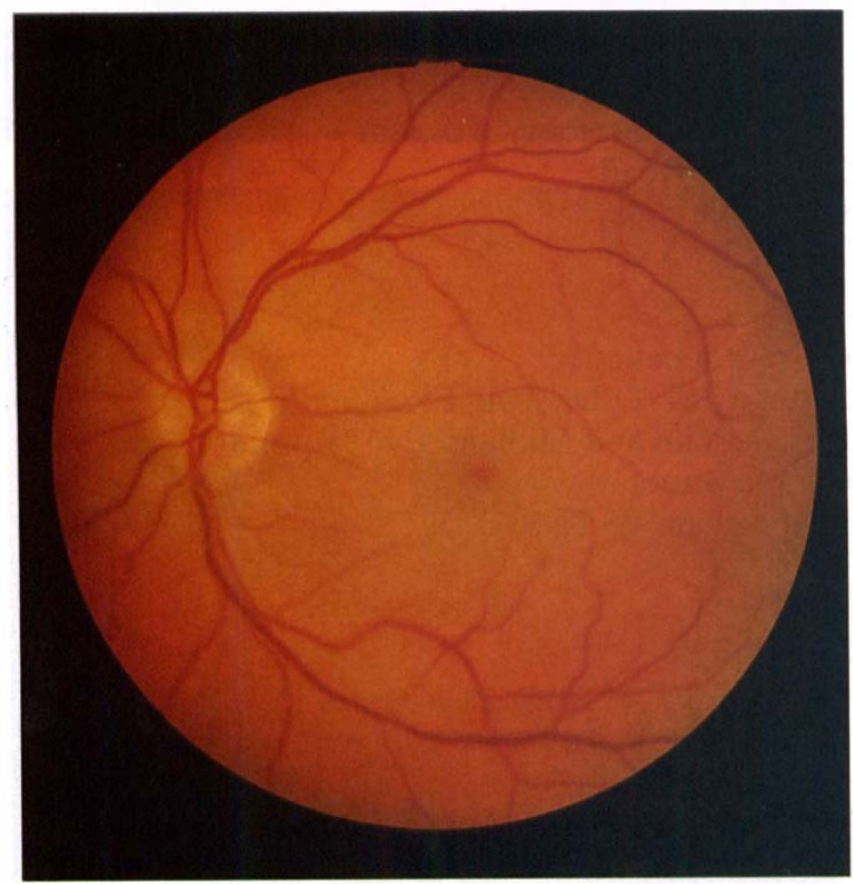

Fig. 1. Retinal photograph of the left eye. Note the central retinal artery occlusion and absence of optic nerve head abnormalities. atrophy was noted in the right eye. Diamox $500 \mathrm{mg}$ intravenously, ocular massage, expired air rebreathing and anterior chamber paracentesis were administered. These measures, however, failed to alleviate his symptoms. He was anticoagulated with $50000 \mathrm{IU}$ heparin subcutaneously and started on $300 \mathrm{mg}$ aspirin o.d..

\section{Investigations}

Full blood count, erythrocyte sedimentation rate, activated partial thromboplastin time and thrombin time were normal; as were euglobulin clot lysis time before and after limb occlusion. Platelet aggregation (on aspirin; with 1 and $5 \mu \mathrm{mol}$ ADP) was normal. Screens for antinuclear antibody, antineutrophil cytoplasmic antibody, antiphospholipid antibodies, rheumatoid factor and Treponema pallidum haemagglutination test were negative. Serum biochemistry and lipid profile showed no abnormality. Doppler carotid ultrasonography was unremarkable and trans-oesophageal echocardiography showed a partial atrial-septal defect but no source for cardiac emboli. An orbital CT scan showed bilateral buried optic nerve drusen (Figs. 2, 3) which were confirmed by ultrasonography.

Doppler ultrasonography demonstrated a central retinal artery blood velocity of $0.03 \mathrm{~m} / \mathrm{s}$ in the right eye and $0.06 \mathrm{~m} / \mathrm{s}$ in the left eye (NR $0.1 \mathrm{~m} / \mathrm{s}$ ). The patient was discharged home and local ophthalmology and medical outpatient appointments were made for immediate support and follow-up.

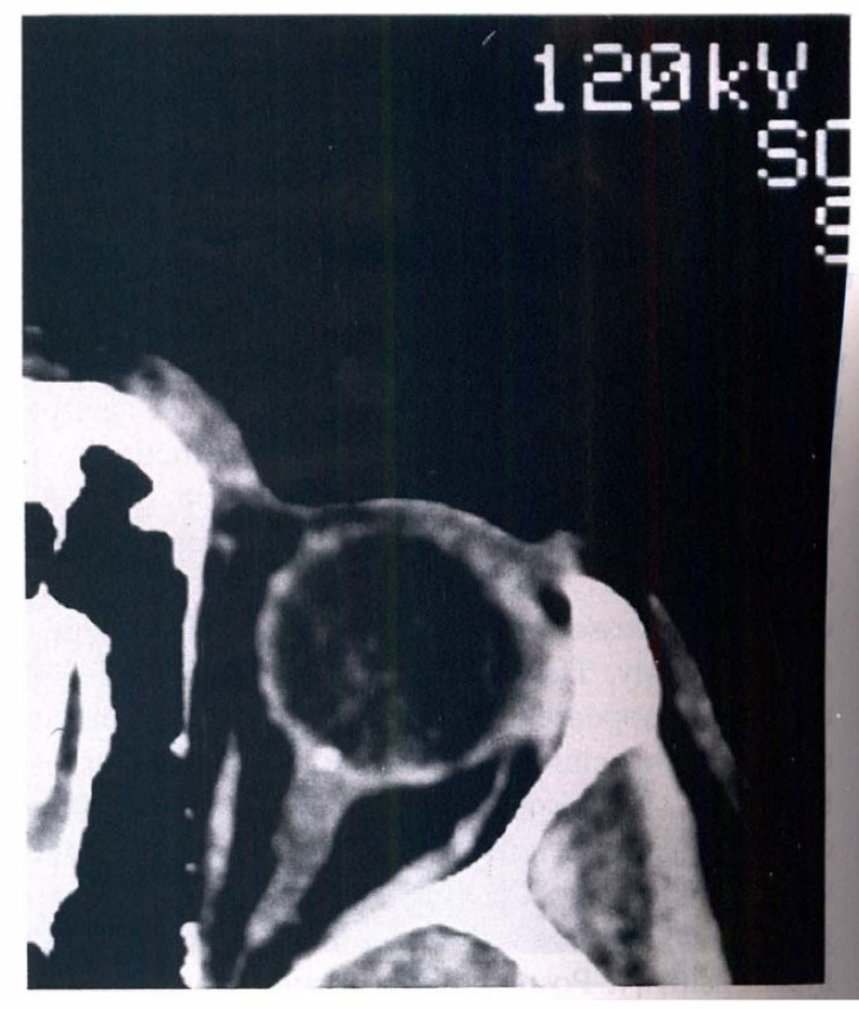

Fig. 2. Orbital CT scan of the right orbit. Note the large buried drusen within the optic nerve. 


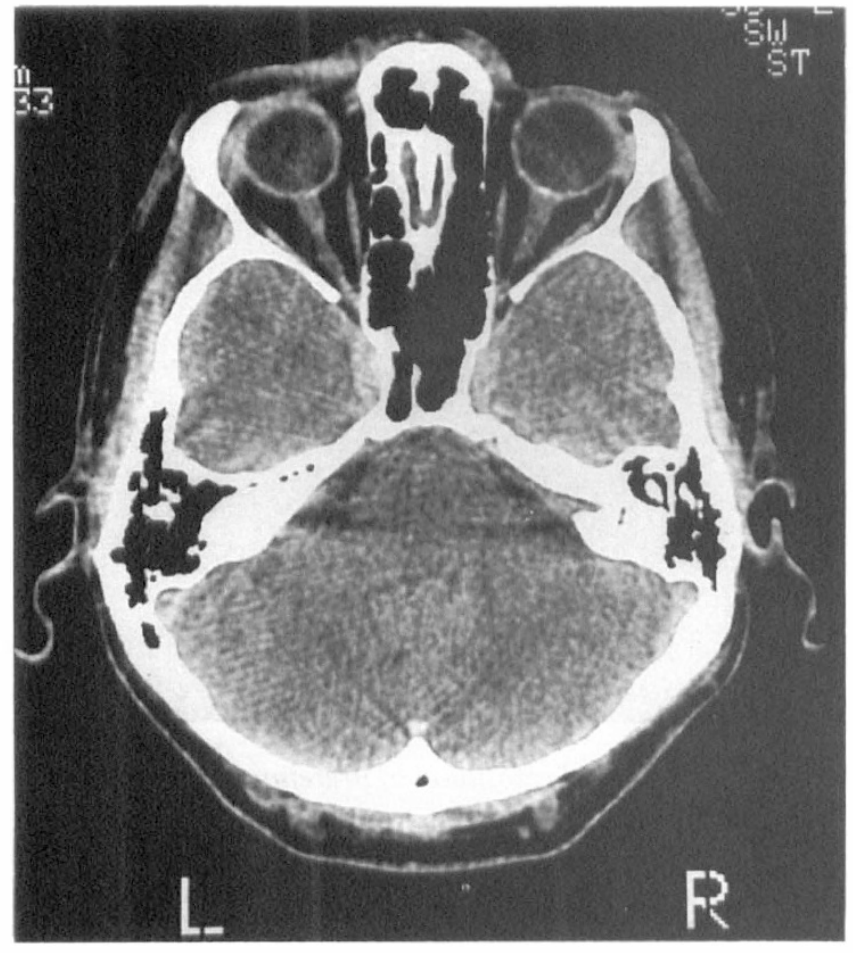

Fig. 3. Orbital CT scan of both orbits demonstrating bilateral disease.

\section{Discussion}

Optic nerve head drusen occur in $1 \%$ of patients; $80 \%$ are bilateral. ${ }^{3}$ The incidence of buried optic nerve head drusen is unknown. They are, however, associated with sudden concentric constriction of the visual field, ${ }^{4}$ blind spot enlargement, ${ }^{5}$ nerve fibre layer defects and splinter, vitreous and subretinal haemorrhages. ${ }^{6}$ They are also associated with central retinal vein occlusion ${ }^{7}$ and anterior ischaemic optic neuropathy in young patients. ${ }^{8}$ Optic disc drusen may affect blood flow to the retina, possibly by compression of vessels. The association between buried drusen and CRAO is not widely recognised but may have a similar pathogenesis.

This patient demonstrated the importance of full investigation of young patients presenting with CRAO. Ocular, carotid, cardiac imaging and involvement of the haematology department are essential. In this case platelet agglutination was normal even in the presence of aspirin. This may have been due to the presence of a slight cardiac abnormality, exciting the platelets and rendering his prophylactic treatment ineffective.

CRAO treatment has limited success. If the main treatments of intravenous Diamox, massage and paracentesis fail to work there are few second-line alternatives. Streptokinase may be of use in retinal thrombolysis; ${ }^{9}$ however, it is associated with a significant rate of morbidity. This man's experience may be relevant for air travellers with retinal vascular disease as both CRAOs occurred on descent from altitude.
Arteries and veins dilate by $7 \%$ and $12 \%$ respectively at atmospheric pressures lower than $565 \mathrm{mmHg}^{10}$ (equivalent to an altitude of $2500 \mathrm{~m}$ ), the cabin pressure commonly used in passenger aircraft. However, there is little information on the effect of a sudden descent on the tone of blood vessels or intraocular pressure. There may be reactive vasoconstriction during descent, triggering occlusion in patients with compromised circulations. Others have shown ischaemic changes in patients exposed to high altitudes ${ }^{1}$ and some even central retinal vein occlusion with vitreous haemorrhage. ${ }^{2}$

This unfortunate man raises questions concerning the appropriate investigation and monitoring for young patients with CRAO and about the wisdom of air travel for patients with retinal vascular disease.

\author{
R. S. B. Newsom \\ Department of Ophthalmology \\ St Thomas' Hospital \\ London, UK \\ D. R. Trew \\ Department of Ophthalmology \\ Queen Mary's Hospital \\ Sidcup, Kent, UK
}

\section{T. J. K. Leonard \\ Department of Ophthalmology \\ Charing Cross Hospital \\ London, UK}

Correspondence to:

Mr R. S. B. Newsom

Department of Ophthalmology

St Thomas' Hospital,

Lambeth Palace Road

London SE1 7EH, UK

\section{References}

1. Butler FK, Harris DJ, Reynolds RD. Altitude retinopathy on Mount Everest, 1989. Ophthalmology 1992; 99:739-46.

2. McFadden DM, Houston CS, Sutton JR, Powles AC, Gray GW, Roberts RS. High-altitude retinopathy. JAMA 1981;245:581-6.

3. Section 5, Neuro-ophthalmology. American Academy of Ophthalmology Basic and Clinical Science Course. San Francisco: American Academy of Ophthalmology, 1944:81.

4. Moody TA, Irvine AR, Cahn PH, Susac JO, Horton JC. Sudden visual field constriction associated with optic disc drusen. J Clin Neuroophthalmol 1993; 13:8-13.

5. Hoover DL, Robb RM, Petersen RA. Optic disc drusen in children. J Pediatr Ophthalmol Strabismus 1988;25:191-5.

6. Ford CS, Biller J, Weaver RG. Drusen-associated visual field defects and hemorrhages. South Med J 1983;76:1060-2. 
7. Chern S, Magargal LE, Annesley WH. Central retinal vein occlusion associated with drusen of the optic disc. Ann Ophthalmol 1991;23:66-9.

8. Gittinger JJ, Lessell S, Bondar RL. Ischemic optic neuropathy associated with optic disc drusen. J Clin Neuroophthalmol 1984;4:79-84.

9. Annonier P, Benichou C, Flament J, Bronner A. [Role of fibrinolysis in the treatment of retinal arterial occlusion: discussion of 5 cases.] Bull Soc Ophtalmol Fr 1988;88:1167-71.

10. Brinchmann HO, Myhre K. Vascular response of retinal arteries and veins to acute hypoxia of 8,000 , $10,000,12,500$ and 15,000 feet of simulated altitude. Aviat Space Environ Med 1990;61:112-6.

Sir,

Valsalva Haemorrhagic Retinopathy in a Pregnant Woman

The term 'Valsalva haemorrhagic retinopathy' describes an entity in which a sudden rise in intrathoracic or intra-abdominal pressure causes a rapid rise of intravenous pressure within the eye with spontaneous rupture of superficial retinal capillaries. Affected patients may give a history of sudden visual loss during or after activities such as heavy lifting, straining at stool, coughing or vomiting. ${ }^{1}$ We report the following case which demonstrates these clinical features.

\section{Case Report}

A 26-year-old woman who was in her second trimester of pregnancy complained of blurred vision a few days after an episode of vomiting. She was otherwise well with no past medical or ocular history

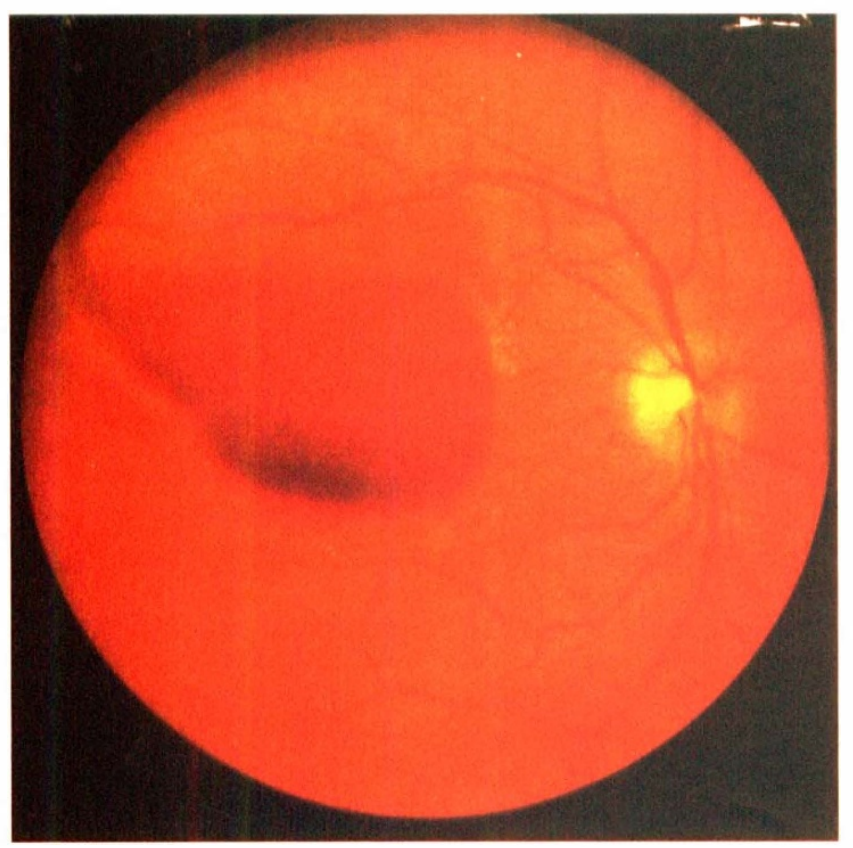

Fig. 1. Large preretinal haemorrhage at macula with fluid level. (Hard exudates at nasal limit of haemorrhage.)

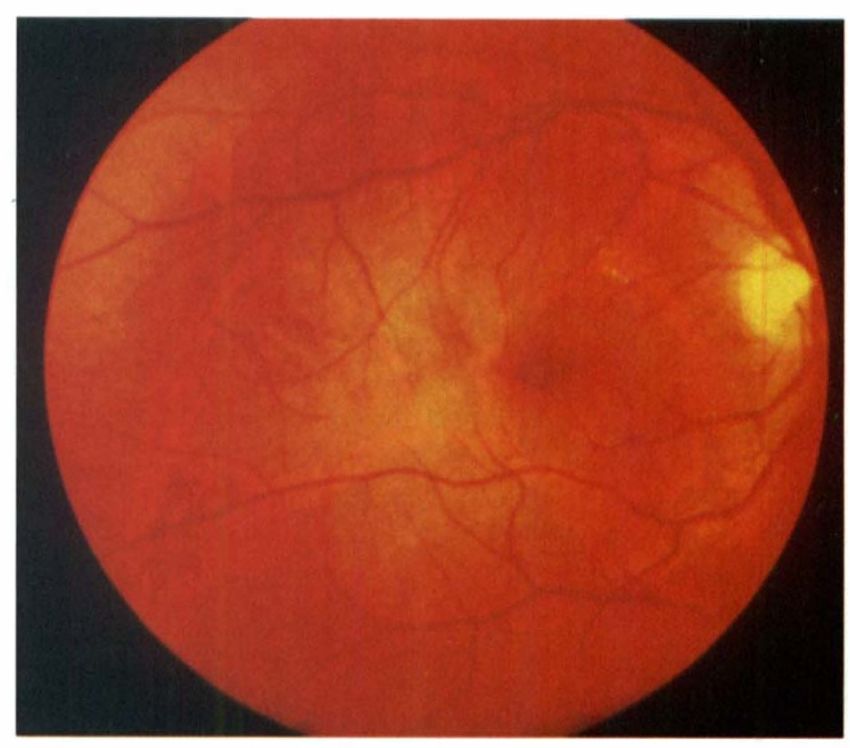

Fig. 2. Almost total resolution of preretinal haemorrhage 6 months later.

of note. Her best corrected visual acuities were counting fingers at $1 \mathrm{~m}$ on the right, $6 / 6$ on the left.

Ocular examination showed normal anterior segments, and a large preretinal haemorrhage at the macula on the right (Fig. 1). Obstetric examination detected no abnormality and coagulation studies were normal.

Management consisted of rest, avoidance of strenuous activities, and regular observation at almost 2 weekly intervals including fundus photography. Spontaneous resolution over a 6 month period was demonstrated (Fig. 2), with a final corrected visual acuity of $6 / 6$. Following delivery of a healthy male infant at full term, a fluorescein angiogram was performed which showed no abnormality (Fig. 3).

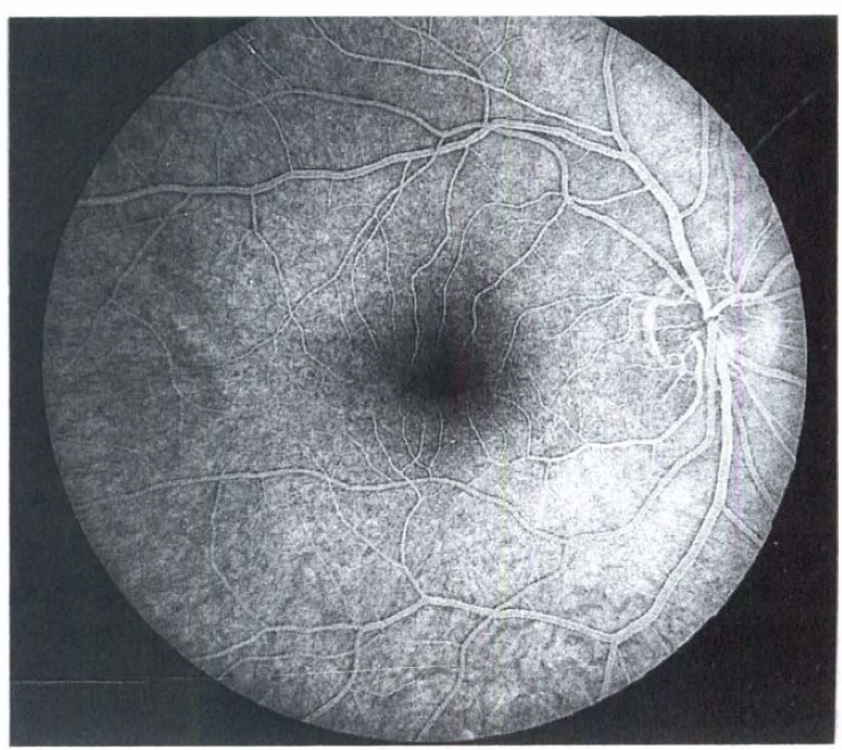

Fig. 3. Mid-venous phase fluorescein angiogram showing normal retinal vasculature. 